\title{
Following Uninsured Patients Through Medicaid Expan- sion: Ambulatory Care Use and Diagnosed Conditions
}

\author{
Nathalie Huguet, $P b D^{1}$ \\ Steele Valenzuela, MS ${ }^{1}$ \\ Miguel Marino, $\mathrm{PbD}^{1,2}$ \\ Heather Angier, $\mathrm{PbD}, \mathrm{MPH}^{1}$ \\ Brigit Hatch, MD, $M P H^{1,3}$ \\ Megan Hoopes, MPH \\ Jennifer E. DeVoe, MD, DPbil \\ 'Department of Family Medicine, \\ Oregon Health \& Science University, \\ Portland, Oregon \\ ${ }^{2}$ Division of Biostatistics, School of Public \\ Health, Oregon Health \& Science Univer- \\ sity, Portland State University, Portland, \\ Oregon
}

${ }^{3}$ Research Department, OCHIN Inc, Portland, Oregon

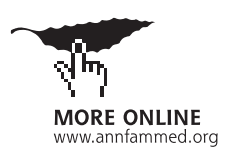

Conflicts of interest: authors report none.

\section{CORRESPONDING AUTHOR}

Nathalie Huguet

Department of Family Medicine

Oregon Health \& Science University

3181 SW Sam Jackson Park Rd

Portland, OR 97239-3098

huguetn@ohsu.edu

\begin{abstract}
PURPOSE The Patient Protection and Affordable Care Act (ACA) has improved access to health insurance, yet millions remain uninsured. Many patients who remain uninsured access care at community health centers (CHCs); however, little is known about their health conditions and health care use. We assessed ambulatory care use and diagnosed health conditions among a cohort of $\mathrm{CHC}$ patients uninsured before enactment of the ACA (pre-ACA: January 1, 2012 to December 31, 2013) and followed them after enactment (post-ACA: January 1, 2014 to December 31, 2015).

METHODS This retrospective cohort analysis used electronic health record data from CHCs in 11 US states that expanded Medicaid eligibility. We assessed ambulatory care visits and documented health conditions among a cohort of 138,246 patients (aged 19 to 64 years) who were uninsured pre-ACA and either remained uninsured, gained Medicaid, gained other health insurance, or did not have a visit post-ACA. We estimated adjusted predicted probabilities of ambulatory care use using an ordinal logistic mixed-effects regression model.
\end{abstract}

RESULTS Post-ACA, $20.9 \%$ of patients remained uninsured, $15.0 \%$ gained Medicaid, $12.4 \%$ gained other insurance, and $51.7 \%$ did not have a visit. The majority of patients had $\geq 1$ diagnosed health condition. The adjusted proportion of patients with high use ( $\geq 6$ visits over 2 years) increased from pre-ACA to post-ACA among those who gained Medicaid (pre-ACA: 23\%, post-ACA: $34 \%$, $P<.001$ ) or gained other insurance (pre-ACA: $29 \%$, post-ACA: 48\%, $P<.001$ ), whereas the percentage fell slightly for those continuously uninsured.

CONCLUSIONS A significant percentage of $\mathrm{CHC}$ patients remained uninsured; many who remained uninsured had diagnosed health conditions, and one-half continued to have $\geq 3$ visits to CHCs. CHCs continue to be essential providers for uninsured patients.

Ann Fam Med 2019;17:336-344. https://doi.org/10.1370/afm.2385.

\section{INTRODUCTION}

I n 2010, the Patient Protection and Affordable Care Act (ACA) was enacted with the goal of expanding health insurance coverage to all US citizens and legal residents. ${ }^{1}$ The ACA improved coverage via several mechanisms including Medicaid eligibility expansion, introduction of health insurance marketplaces, and a mandate for individuals to obtain coverage. Subsequently, the number of uninsured individuals dropped from 43 million in 2013 to 27 million in $2016 .{ }^{2}$ Self-reported data suggest that those who remain uninsured post-ACA are disproportionally Hispanic, aged less than 35 years, and from low-income households. ${ }^{3}$ Affordability of health insurance plans and eligibility to qualify for Medicaid or tax credits are the most common barriers to gaining insurance. ${ }^{3}$

Community health centers $(\mathrm{CHCs})$ provide services for many uninsured individuals; they are essential safety-net providers that serve nearly 28 million people yearly. A large proportion of patients seen at $\mathrm{CHC}$ s have low incomes, with $85 \%$ at or below $150 \%$ of the federal poverty level (FPL). Community health centers provide health care services by reducing barri- 
ers to cost, accepting uninsured patients, and tailoring services for vulnerable populations (eg, homeless, nonEnglish speakers). ${ }^{4}$ The ACA augmented funding for CHCs for 5 years (through 2017), which was renewed in early 2018, allowing them to continue to provide care for the uninsured, assist insured patients with significant out-of-pocket expenses, and provide services not covered by health insurance. ${ }^{5,6}$ Post-ACA, CHCs experienced an increase in both Medicaid-paid visits and privately insured visits ${ }^{6-10}$ and corresponding decreases in uninsured visits. More than 6 million patients seen at CHCs across the nation, however, remain uninsured. ${ }^{4}$

With increasing demands from privately insured patients experiencing difficulties with out-of-pocket expenses 5 and from the growing numbers of Medicaid beneficiaries, $\mathrm{CHC}$ s report increased wait times for appointments, ${ }^{5}$ which may affect access to care for patients who remain uninsured. Primary care use among $\mathrm{CHC}$ patients who remained uninsured postACA, however, is largely unknown. A recent survey of patients seen at CHCs found that those who were uninsured were more likely to report difficulty accessing care than insured patients, though it is unclear from these data if the barriers were more or less prevalent post-ACA because they did not compare these post-ACA findings to the pre-ACA period. ${ }^{11}$

In the present study, we followed a cohort of $\mathrm{CHC}$ patients who were uninsured before ACA enactment and remained uninsured or gained insurance post-ACA and used electronic health record (EHR) data to assess diagnosed health conditions, characterize how much and what type of ambulatory care was received, and compare patterns of ambulatory care use before and after ACA Medicaid expansion.

\section{METHODS}

\section{Data}

This study was approved by our institution's Institutional Review Board. We used EHR data from the Accelerating Data Value Across a National Community Health Center Network (ADVANCE) clinical data research network (CDRN) of the National PatientCentered Clinical Research Network (PCORnet). ${ }^{12}$ The ADVANCE CDRN is a unique community laboratory for research on underrepresented populations receiving care at $\mathrm{CHCs}$. The study time period spanned 4 years; 2 years before the implementation of ACA insurance expansions (pre-ACA: January 1, 2012 to December 31, 2013) and 2 years after (post-ACA: January 1, 2014 to December 31, 2015). The study sample included all nonpregnant patients without insurance pre-ACA, aged 19 to 64 years during the study period, with $\geq 1$ ambulatory visit pre-ACA to $\geq 1$ of 300 primary care CHCs and who were included in their EHR system as of January 1, 2012 in 11 states that expanded Medicaid eligibility after ACA implementation (California, Hawaii, Maryland, Minnesota, Nevada, New Mexico, Ohio, Oregon, Rhode Island, Washington, and Wisconsin). We restricted the study to patients residing in expansion states because (1) a large number of $\mathrm{CHC}$ patients became eligible for Medicaid and would only have the option to apply in states that expanded, (2) unlike private insurance, Medicaid premiums and cost-sharing amounts, if charged, are capped ${ }^{13}$ allowing for more control over changes in health care use related to ACA Medicaid expansion, and (3) federal law requires that Medicaid provide coverage for specified services, ${ }^{14}$ allowing for comparability of services received. We excluded pregnant women because there is a Medicaid program that covers low-income pregnant women not otherwise eligible for Medicaid, and they have very different usage patterns and health care needs than nonpregnant women. We followed 138,246 patients who were uninsured at all visits before ACA Medicaid expansion (representing $28 \%$ of the patient population aged 19 to 64 years) and categorized them into 4 mutually exclusive post-ACA insurance coverage groups (described below).

\section{Measures}

\section{Insurance Coverage}

Electronic health record data contain information on payer types as well as billable codes for services performed at each ambulatory care visit. Because these data are used for billing purposes, they represent reliable information on insurance status and services received at each visit. ${ }^{15}$ The mutually exclusive post-ACA insurance coverage groups ${ }^{16}$ included the following:

- Continuously Uninsured ( $\mathrm{n}=28,834)$ : all preperiod visits and all postperiod visits were uninsured.

- Gained Medicaid ( $n=20,766$ ): all preperiod visits were uninsured, and all postperiod visits were covered by Medicaid with the following exception: to allow for time to enroll in Medicaid, the first visit post-ACA could be uninsured $(n=3,052 ; 14.7 \%$ of the group).

- Gained Other Insurance ( $\mathrm{n}=17,171)$ : all preperiod visits were uninsured, and during the post-ACA period, visits were covered by other insurance types (private: $\mathrm{n}=3,690$; other public: $\mathrm{n}=232$ ) or insurance patterns (had privately insured visit followed by uninsured visit[s]: $n=3,257$; had Medicaid-insured visit followed by uninsured visit[s]: $\mathrm{n}=5,834$; other patterns: $\mathrm{n}=4,158$ ).

We also examined those who were uninsured pre-ACA and had no visits in the post-ACA period (No PostACA Period Ambulatory Care Visits group $[\mathrm{n}=71,475])$. 


\section{Ambulatory Care Use Outcomes}

The numbers and types of $\mathrm{CHC}$ ambulatory care visits were determined using the primary Current Procedural Terminology code for each visit. Overall ambulatory care use included total visits (numbers of all billable encounters including primary care, preventive care, mental care, etc), primary care visits (all primary care visits), and receipt of preventive care services (nonproblem-focused encounters for general wellness and prevention). We computed visit rates by dividing the number of visits in a given interval (ie, pre- or postACA period) by the total number of adult patients seen at a clinic over the study period, interpreted as the average number of visits per patient per period. To assess the effect of ACA Medicaid expansion among high, medium, and low users, visits were categorized as 0 (for primary or preventive care only), 1 to 2,3 to 5 , and $\geq 6$ visits over 2 years in both the pre- and postACA periods; we considered $\geq 6$ visits high use. Visit types were considered to be primary care or preventive services. These categories were adapted from previous studies. ${ }^{17,18}$ We collapsed the 6 -to-10- and $>10$-visit categories from prior studies into 1 category of $\geq 6$ visits for ease of interpretation and to have enough samples in the categories to reliably model this as an outcome.

\section{Health Condition Outcomes}

Clinical complexity was measured using the revised Charlson Comorbidity Index. This revised version of the index supplements the prior validated index as a predictor of costs of chronic disease for patients in primary care, with additional weights for mental and behavioral health conditions. ${ }^{19}$

Diagnosed health conditions were identified according to the International Classification of Diseases, Ninth or Tenth Revision codes on patients' problem lists or encounter diagnoses. We selected a subset of health conditions fitting the category of a preexisting condition on the basis of the Kaiser Family Foundation's list of declinable medical conditions; those that would have likely rendered the patient uninsurable before passage of the ACA. ${ }^{20}$ These included human immunodeficiency virus infection/acquired immune deficiency syndrome; systemic lupus erythematosus; alcohol or drug abuse, mental disorders (eg, bipolar disorder) Alzheimer's disease/dementia ${ }_{i}$ multiple sclerosis; rheumatoid arthritis, fibromyalgia, or other inflammatory joint disease; muscular dystrophy; cancer other than skin, severe obesity; cerebral palsy; ${ }_{i}$ congestive heart failure, paraplegia or paralysis; coronary artery disease; Crohn's disease or ulcerative colitis; Parkinson's disease ${ }_{i}$ chronic obstructive pulmonary disease, emphysema, or asthma; diabetes mellitus; Pneumocystis pneumonia epilepsy; hemophilia; sleep apnea; hepatitis; stroke $_{i}$ and kidney disease/renal failure. We assessed the number of conditions in the pre-ACA period and newly diagnosed conditions in the post-ACA period.

\section{Patient Characteristics}

Patient characteristics included sex, age group (1926, 27-44, 45-64 years), race/ethnicity (non-Hispanic white, non-Hispanic black, Hispanic, non-Hispanic other), preferred language (English, Spanish, other), FPL $(\leq 138 \%,>138 \%)$, and urban/rural clinic location.

\section{Statistical Analyses}

We computed descriptive statistics to compare the demographic characteristics of patients in each insurance group. In addition, for each insurance cohort, we estimated the average number of diagnosed health conditions and the Charlson Comorbidity Index scores for the pre- and post-ACA periods.

To assess changes in patterns of ambulatory care use from pre-ACA to post-ACA, we analyzed numbers of visits in 2 ways as follows: (1) by treating the visit count as a discrete variable (eg, 1, 2, ..etc, visits per period) and (2) as an ordinal categorical variable (1-2, $3-5$, and $\geq 6$ visits per period). The first approach provides overall ambulatory care use rates over time by group, whereas the second approach provides insights into whether insurance groups have heterogeneous effects on whether pre-ACA high/medium/low users are maintaining similar usage breakdowns after ACA expansion. For the discrete visit outcome, we used a Poisson mixed-effects model. For the ordinal categorical visit outcome, we used an ordinal logistic mixed-effects model and estimated the adjusted predicted probability of categorical ambulatory care use (ie, having 1-2, 3-5 or $\geq 6$ visits over 2 years) for each ACA period. For both models, we accounted for clustering of pre- and postperiod visits by patients and patients by clinics via the following 2 random effects: a random intercept at the $\mathrm{CHC}$ level and a random intercept at the patient level. The fixed-effects portion of the models included insurance group, an indicator for pre-ACA or post-ACA period, an interaction between insurance group and the period indicator, age, race/ethnicity, primary language, sex, FPL, urban/rural status, and Charlson Comorbidity Index score. Data management and analyses were conducted using R Version 3.5.0 (R Project for Statistical Computing) and Stata 15 (StataCorp LLC). Statistical significance was set at a type I error of $5 \%$.

\section{RESULTS}

Table 1 summarizes the demographic characteristics of patients stratified by post-ACA Medicaid expansion insurance. Among patients who were uninsured before 
the ACA, 20.9\% remained continuously uninsured, $15.0 \%$ gained Medicaid, $12.4 \%$ gained other insurance, and $51.7 \%$ did not have an ambulatory care visit during the 2-year post-ACA period. Those who were continuously uninsured were more likely to be aged 27 to 44 years, Hispanic, and speak Spanish as their preferred language compared to the other insurance groups. Patients who gained Medicaid after enactment of the ACA were more likely to be aged 45 to 64 years, nonHispanic white, and to speak English as their preferred language. Those with no postperiod ambulatory care visits were more likely to be aged 19 to 26 years and male compared to the other insurance groups. Among patients who gained other insurance or had inconsistent coverage patterns, a large proportion were aged 45 to
64 years, female, Hispanic, and had Spanish as their preferred language compared to the other insurance groups. Of the 4 post-ACA insurance groups, those who gained other insurance had the greatest share of patients with incomes $>138 \%$ FPL. Lastly, more than $60 \%$ of patients in all 4 post-ACA insurance groups had incomes $\leq 138 \%$ FPL, the cut-point for Medicaid eligibility, and most received care in urban clinics.

Regarding health status, patients who gained Medicaid or gained other insurance had greater clinical complexity (greater Charlson Comorbidity Index scores) than those continuously uninsured or those who did not have postperiod ambulatory care visits (Table 2). Among patients with post-ACA visits, the Charlson Comorbidity Index score remained higher

Table 1. Characteristics of the Community Health Center Patients Who Were Uninsured Before the ACA, Stratified by Insurance Group ( $=138,246)$

\begin{tabular}{|c|c|c|c|c|}
\hline & $\begin{array}{c}\text { Continuously } \\
\text { Uninsured }^{\mathrm{a}} \\
(\mathrm{n}=28,834 \\
[20.9 \%])\end{array}$ & $\begin{array}{c}\text { Gained Medicaid } \\
\text { (n = 20,766 [15.0\%]) }\end{array}$ & $\begin{array}{c}\text { Gained Other } \\
\text { Insurance } \\
\text { (n=17,171 } \\
[12.4 \%])\end{array}$ & $\begin{array}{l}\text { No Post-ACA Period } \\
\text { Ambulatory Care Visits } \\
(\mathbf{n}=71,475[51.7 \%])\end{array}$ \\
\hline \multicolumn{5}{|l|}{ Sex, No. (\%) } \\
\hline Female & $15,607(54.1)$ & $10,694(51.5)$ & $9,958(58.0)$ & $33,128(46.3)$ \\
\hline Male & $13,227(45.9)$ & $10,072(48.5)$ & $7,213(42.0)$ & $38,347(53.7)$ \\
\hline \multicolumn{5}{|c|}{ Age during study period, No. (\%), y } \\
\hline $19-26$ & $4,890(17.0)$ & $3,279(15.8)$ & $2,277(13.3)$ & $18,301(25.6)$ \\
\hline $27-44$ & $15,303(53.1)$ & $8,232(39.6)$ & $6,937(40.4)$ & $32,217(45.1)$ \\
\hline $45-64$ & $8,641(30.0)$ & $9,255(44.6)$ & $7,957(46.3)$ & $20,957(29.3)$ \\
\hline \multicolumn{5}{|l|}{ Race/ethnicity, No. (\%) } \\
\hline Non-Hispanic white & $8,706(30.2)$ & $10,596(51.0)$ & $6,984(40.7)$ & $31,383(43.9)$ \\
\hline Hispanic & $16,288(56.5)$ & $5,867(28.2)$ & $6,808(39.6)$ & $22,516(31.5)$ \\
\hline Non-Hispanic black & $2,236(7.8)$ & $2,693(13.0)$ & $2,395(13.9)$ & $10,224(14.3)$ \\
\hline Non-Hispanic other & $608(2.1)$ & $918(4.4)$ & $513(3.0)$ & $2,637(3.7)$ \\
\hline Missing & $996(3.5)$ & $692(3.3)$ & $471(2.7)$ & $4,715(6.6)$ \\
\hline \multicolumn{5}{|c|}{ Preferred language, No. (\%) } \\
\hline English & $14,487(50.2)$ & $16,710(80.5)$ & $12,036(70.1)$ & $54,886(76.8)$ \\
\hline Spanish & $13,512(46.9)$ & $3,124(15.0)$ & $4,653(27.1)$ & $12,728(17.8)$ \\
\hline Other & $505(1.8)$ & $842(4.1)$ & $399(2.3)$ & $2,223(3.1)$ \\
\hline Missing & $330(1.1)$ & $90(0.4)$ & $83(0.5)$ & $1,638(2.3)$ \\
\hline \multicolumn{5}{|c|}{ Federal poverty level, No. (\%) } \\
\hline$\leq 138 \%$ & $18,780(65.1)$ & $17,034(82.0)$ & $10,978(63.9)$ & $50,761(71.0)$ \\
\hline$>138 \%$ & $4,608(16.0)$ & $2,085(10.0)$ & $3,861(22.5)$ & $9,469(13.2)$ \\
\hline Missing & $5,446(18.9)$ & $1,647(7.9)$ & $2,332(13.6)$ & $11,245(15.7)$ \\
\hline \multicolumn{5}{|l|}{ Clinic location, No. (\%) } \\
\hline Rural & $1,736(6.0)$ & $1,983(9.5)$ & $2,304(13.4)$ & $6,000(8.4)$ \\
\hline Urban & $27,098(94.0)$ & $18,783(90.5)$ & $14,867(86.6)$ & $65,475(91.6)$ \\
\hline \multicolumn{5}{|c|}{ ACA = Patient Protection and Affordable Care Act. } \\
\hline \multicolumn{5}{|c|}{$\begin{array}{l}\text { Notes: Pre-ACA = January 1, } 2012 \text { to December 31, 2013; Post-ACA = January 1, } 2014 \text { to December 31, 2015. Visits refer to ambulatory care only. Pairwise insurance } \\
\text { group differences for Continuously Uninsured vs Gained Medicaid, Continuously Uninsured vs Gained Other Insurance, and Continuously Uninsured vs No Post-ACA } \\
\text { Period Ambulatory Care Visits were statistically significant }(P<.05) \text {. }\end{array}$} \\
\hline \multicolumn{5}{|c|}{ a All preperiod visits were uninsured, and all postperiod visits were uninsured. } \\
\hline \multicolumn{5}{|c|}{$\begin{array}{l}{ }^{b} \text { All preperiod visits were uninsured, and all postperiod visits were covered by Medicaid with the following exception: to allow for time to enroll in Medicaid, the first } \\
\text { visit post-ACA could be uninsured. }\end{array}$} \\
\hline
\end{tabular}


for patients who gained Medicaid or other insurance compared to those who were continuously uninsured More than $50 \%$ of the patients with postperiod visits had $\geq 1$ diagnosed health condition, that is, health conditions on the list of preexisting conditions often used by insurers pre-ACA to deny coverage, in the pre-ACA period. In the post-ACA period, those who gained Medicaid or other insurance had a diagnosis of, on average, 1 additional condition. Those with no ambulatory care visits in the post-ACA period had the fewest average number of diagnosed health conditions Importantly, by the end of the study period, excluding those with no postperiod ambulatory care visits, all groups averaged $\geq 2$ diagnosed health conditions.

\section{Ambulatory Care Use}

Table 3 summarizes primary and preventive ambulatory care usage patterns pre- and post-ACA by insurance group. The unadjusted overall visit rate before the ACA was similar between those continuously insured and those who gained Medicaid or other insurance and was lowest among patients who did not have a post-ACA visit. Post-ACA, the unadjusted overall visit rate among those who gained Medicaid or other insurance increased significantly, whereas the visit rate decreased among those continuously uninsured (see
Supplemental Appendix 1, http://www.annfammed.org/ content/17/4/336/suppl/DC1 for full model results of adjusted visit rates). Less than $25 \%$ of patients with no postperiod visit had $\geq 3$ visits in the 2 years before ACA initiation compared to more than $50 \%$ of patients in the other 3 groups (continuously uninsured, gained Medicaid, or gained other insurance). During the post-ACA period, those who gained Medicaid or other insurance had greater usage ( $\geq 6$ visits) relative to the continuously uninsured group. Nonetheless, $50 \%$ of those who were continuously uninsured had $\geq 3$ visits during the post-ACA period, and $22 \%$ had $\geq 6$ visits. As shown in Table 3, most of these visits were primary care visits. More than $80 \%$ of uninsured patients in the pre-ACA period had no preventive care visits. In the post-ACA period, there was a drop in the proportion of preventive care visits among those who were continuously uninsured (pre-ACA $=16.0 \%$ and post- $\mathrm{ACA}=13.9 \%$ ). In contrast, those who gained Medicaid insurance (pre$\mathrm{ACA}=10.9 \%$ and post- $\mathrm{ACA}=18.5 \%$ ) or other insurance (pre-ACA $=14.5 \%$ and post-ACA $=24.9 \%$ ) showed significant increases in preventive care visits post-ACA All within- (pre- vs post-ACA) and between-group differences were significant $(P<.05)$.

We assessed whether patients who gained Medicaid or other insurance were more (or less) likely to increase

Table 2. Pre- and Post-ACA Diagnosed Health Conditions and Clinical Complexity of Community Health Center Patients Who Were Uninsured in the Pre-ACA Period, Stratified by Insurance Group $(\mathrm{N}=138,246)$

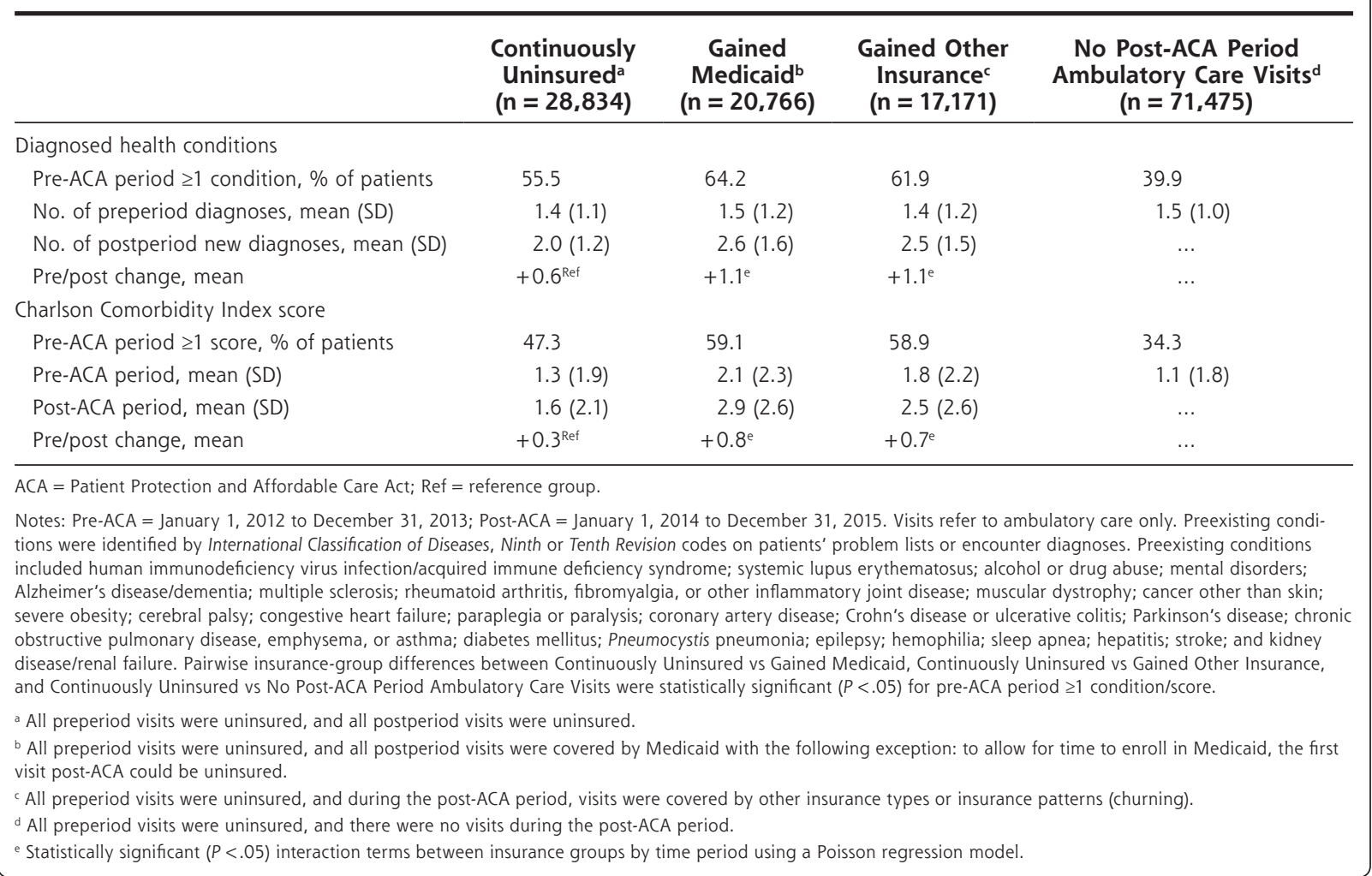


their visit frequency after implementation of the ACA compared to those who remained uninsured, while controlling for important confounders. Figure 1 shows the ordinal logistic mixed-effects regression predicted probabilities of visit frequency $(1-2,3-5$, or $\geq 6$ visits) in the pre- and post-ACA periods by insurance group (see

Table 3. Percentage of Ambulatory Primary and Preventive Care Visits Stratified by Insurance Group and ACA Period

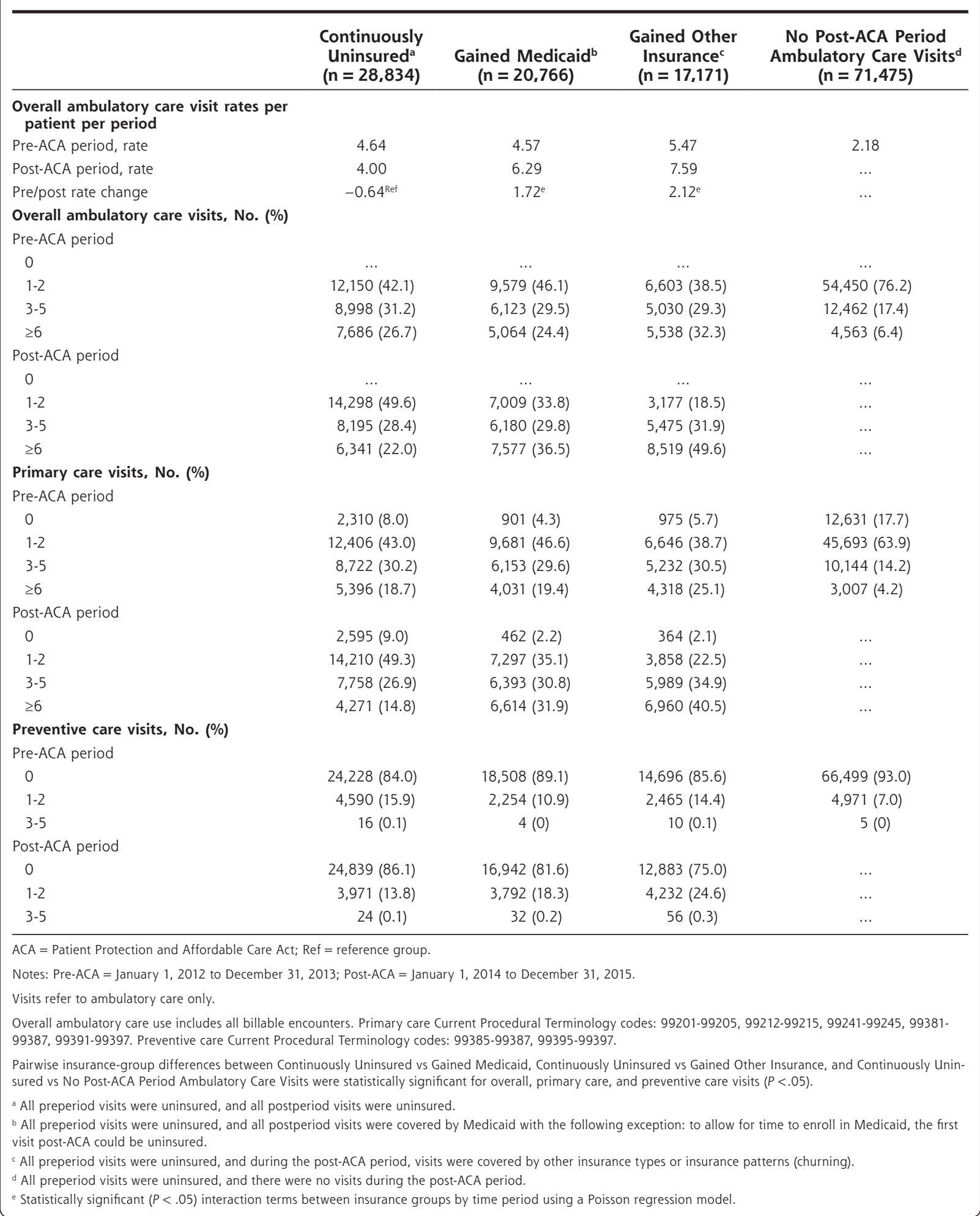


Figure 1. Predicted probabilities of categorizing to 1 of 3 post-ACA visit levels $(1-2,3-5, \geq 6$ overall ambulatory care visits per period) by insurance group and period.

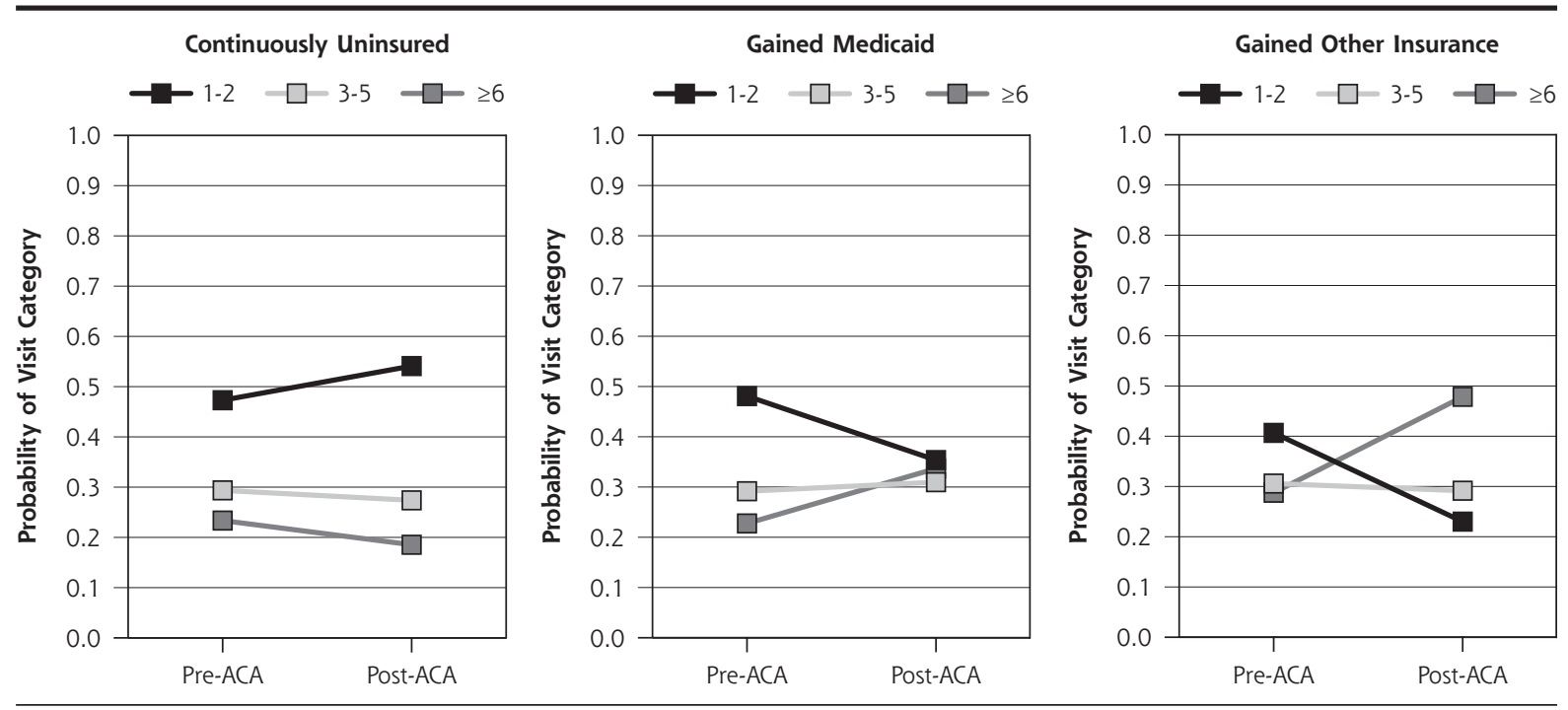

ACA = Patient Protection and Affordable Care Act.

Notes: Pre-ACA = January 1, 2012 to December 31, 2013; Post-ACA = January 1, 2014 to December 31, 2015.

Visits refer to overall ambulatory care use, which includes all billable encounters.

Continuously Uninsured: All preperiod visits were uninsured, and all postperiod visits were uninsured.

Gained Medicaid: All preperiod visits were uninsured, and all postperiod visits were covered by Medicaid with the following exception: to allow for time to enroll in Medicaid, the first visit post-ACA could be uninsured.

Gained Other Insurance: All preperiod visits were uninsured, and during the post-ACA period, visits were covered by other insurance types or insurance patterns (churning).

Pre/post changes in the predicted probabilities were significantly different for each insurance group $(P<.05)$ after adjusting for age, sex, language, race/ethnicity, federal poverty level, clinic location, and Charlson Comorbidity Index score (see Supplemental Appendix 2, http://www.annfammed.org/content/17/4/336/suppl/DC1 for full model results).

Supplemental Appendix 2, http://www.annfammed. org/content/17/4/336/suppl/DC1 for full model results). These results show that among those who remained uninsured, the probability of having 1 to 2 visits increased significantly from pre-ACA to post-ACA ( $47 \%$ to $54 \%$; $P<.001$ ), whereas it decreased for those who gained Medicaid (pre-ACA: $48 \%$, post-ACA: $35 \% ; P<.001)$ or gained other insurance (pre-ACA: $41 \%$, post-ACA: $23 \% ; P<.001)$. Among patients who gained any insurance, there was a significant increase in the probability of having $\geq 6$ visits (gained Medicaid [pre-ACA: $23 \%$, post-ACA: $\left.34 \%_{i} P<.001\right]_{i}$ gained other insurance [pre-ACA: 29\%, post-ACA: $48 \%$; $P<.001])$. This pattern, however, was not observed among patients who remained continuously uninsured.

\section{DISCUSSION}

After ACA Medicaid expansion, a sizable proportion of established patients seen at Medicaid expansion state $\mathrm{CHC}$ remained uninsured. As part of ACA reform, many $\mathrm{CHC}$ s received funding from the Health Resources and Services Administration, which was aimed at providing assistance, such as help with applying for Medicaid coverage, for patients without insurance. Those who remain uninsured at CHCs appear to be unable to take advantage of these services or the increased insurance opportunities provided by the ACA. In the present study, one-half of those patients who remained continuously uninsured were Hispanic and may not be eligible to participate in the Medicaid program, which is restricted to US citizens and lawful immigrants. In addition, many uninsured patients are unaware or do not understand the complex eligibility rules for Medicaid, enrollment periods for marketplace coverage, and/or definitions of deductibles, premiums, and benefits covered. ${ }^{21-23}$ The recent removal of the individual mandate penalty and the shortening of the enrollment time for marketplace insurance is expected to increase the numbers of uninsured. Additional funding to support $\mathrm{CHC}$ s will be needed for them to continue to provide care for increasing numbers of uninsured patients.

Although prior studies have shown that the ACA improved access to health insurance and decreased uninsurance rates, ${ }^{6-10}$ none have examined changes in 
ambulatory care use among those who remained uninsured, especially among high-use CHC patients. The present study highlights the fact that a large share of patients who remained uninsured had $\geq 3$ primary care visits during the 2 years after ACA Medicaid expansion and that 1 in 5 patients who remained uninsured had $\geq 6$ overall ambulatory care visits. The majority of those who remained uninsured had a diagnosed health condition requiring continuous care for management and to avoid disease complications.

From the pre-ACA to the post-ACA period, a greater percentage of newly insured patients had high use ( $\geq 6$ visits), whereas a smaller percentage of continuously uninsured patients had $\geq 6$ visits. The most likely explanation for this finding is that previously uninsured patients who gained Medicaid or other insurance had unmet health care needs requiring more $\mathrm{CHC}$ visits to address their needs. Prior studies have shown that when $\mathrm{CHC}$ patients have health insurance, they have more visits and can access more services. ${ }^{24}$ This result could also be a statistical phenomenon of regression to the mean. Future research is needed to further investigate and explain this finding.

We also found an increase in the proportion of patients with preventive care visits in the post-ACA period versus the pre-ACA period among those who gained coverage via Medicaid or other insurance but not among those who remained uninsured. This could be explained by the ACA provisions that required all payers to provide full coverage for many preventive screenings. Thus, those who gained insurance could more easily access these preventive care services in the post-ACA period, whereas patients who remained uninsured continued to face barriers, especially with respect to preventive services not provided at most CHC sites (eg, mammograms, colonoscopies).

Approximately one-half of patients who were uninsured pre-ACA did not have ambulatory care visits in the 2-year post-ACA period. Although our data cannot determine whether these patients received care outside of $\mathrm{CHCs}$, these patients had fewer documented health conditions and may not have needed care during the post-ACA period. This number is comparable to that of a previous study reporting that at least $36 \%$ of established $\mathrm{CHC}$ patients did not have a visit within a 2 -year period in the pre-ACA era. ${ }^{16}$ It is possible that these patients remained uninsured, gained insurance and sought care elsewhere, or may have chosen not to seek care due to myriad factors known to be barriers to health care use. However, because most of these patients $(71 \%)$ had an FPL $\leq 138 \%$ in the present study, it is likely that many remained uninsured and/or did not seek care due to affordability. Future research involving mixed- methods approaches should be undertaken to understand the complex barriers encountered by patients when choosing and accessing ambulatory care.

The present study has several limitations. First, by using EHR data from CHCs, we did not report on care received elsewhere. Prior studies have shown, however, that CHCs were more likely than other primary care clinicians to accept patients with Medicaid ${ }^{25}$; therefore, it is unlikely that the patients in the present study received a significant amount of ambulatory care outside of CHCs. Second, for patients with no postperiod visits, we do not know if individuals had insurance and/ or received care outside of CHCs. We suspect many remained uninsured, and consequently the number of those continuously uninsured in the present study was likely underestimated. In addition, our data do not provide information regarding access barriers (eg, transportation, CHC resources, appointment availability) that may have affected patients' ability to seek care beyond health insurance coverage. Third, although we could identify the care received by uninsured patients, we could not determine if recommended ambulatory care was delayed or if all needed care was received, both of which might have affected the frequency of visits. Fourth, analyses were based on data from CHCs in 11 states that expanded Medicaid eligibility and may not be generalizable to all US states or patient populations.

\section{CONCLUSION}

The results of the present study showed that a large proportion of $\mathrm{CHC}$ patients gained insurance coverage, yet many did not benefit from the ACA insurance expansions, suggesting that the ACA did a good job extending coverage but did not provide coverage for all as it was intended to do. With the recent proposed changes to the ACA (eg, alteration of the preexisting condition provision and/or Medicaid eligibility or work requirement), more patients are at risk of becoming uninsured once again. These potential revisions to the ACA could greatly affect CHCs' ability to serve socioeconomically disadvantaged patient populations. Rather than limiting insurance options and coverage, policy makers could focus on understanding and removing the remaining barriers to access to care and simplifying enrollment eligibility. CHCs provide highquality care at reduced cost, and policy makers could learn to promote access to care for all and reduce overall health care expenditures by CHCs.

To read or post commentaries in response to this article, see it online at http://www.AnnFamMed.org/content/17/4/336.

Submitted August 30, 2018; submitted, revised, January 15, 2019; accepted February 28, 2019. 
Key words: medically uninsured; Patient Protection and Affordable Care Act; Medicaid; health care delivery; access to health care; preexisting condition; primary health care

Funding support: This work was supported by the Agency for Healthcare Research and Quality (R01HS024270), the National Cancer Institute (R01CA204267 and R01CA181452), and the National Heart, Lung, and Blood Institute (R01HL136575). The Accelerating Data Value Across a National Community Health Center Network (ADVANCE) is led by the OCHIN Community Health Information Network, in partnership with the Health Choice Network, Fenway Health, CareOregon, Kaiser Permanente Center for Health Research, Legacy Health, Oregon Health \& Science University, and the Robert Graham Center.

Acknowledgments: The authors acknowledge the participation of our partnering health systems.

Disclaimer: The views presented in this article are solely the responsibility of the authors and do not necessarily represent the views of the funding agencies.

Supplementary materials: Available at http://www.AnnFamMed. org/content/17/4/336/suppl/DC1/.

\section{References}

1. Henry J. Kaiser Family Foundation. Summary of the Affordable Care Act. Menlo Park, CA: Henry J. Kaiser Family Foundation; 2013.

2. Henry J Kaiser Familty Foundation. The uninsured: a primer - key facts about health insurance and the uninsured in the wake of national health reform. https://www.kff.org/uninsured/report/ the-uninsured-a-primer-key-facts-about-health-insurance-and-theuninsured-in-the-wake-of-national-health-reform/. Published Nov 1, 2016. Accessed Nov 30, 2017.

3. Collins SR, Gunja MZ, Doty MM, Beutel S. Who are the remaining uninsured and why haven't they signed up for coverage? Issue Brief (Commonw Fund). 2016;24:1-20.

4. National Association of Community Health Centers. Community Health Center Chartbook. Bethesda, MD: National Association of Community Health Centers; 2018.

5. Rosenbaum S, Paradise J, Markus A, et al. Community Health Centers: Recent Growth and the Role of the ACA. Issue Brief. Menlo Park, CA: Henry J. Kaiser Family Foundation; Jan 2017.

6. Han X, Luo Q, Ku L. Medicaid expansion and grant funding increases helped improve community health center capacity. Health Aff (Millwood). 2017;36(1):49-56.

7. Angier $H$, Hoopes $M$, Gold $R$, et al. An early look at rates of uninsured safety net clinic visits after the Affordable Care Act. Ann Fam Med. 2015;13(1):10-16.

8. Hoopes MJ, Angier H, Gold R, et al. Utilization of community health centers in Medicaid expansion and nonexpansion states, 2013-2014. J Ambul Care Manage. 2016;39(4):290-298.

9. Huguet N, Hoopes MJ, Angier H, Marino M, Holderness H, DeVoe JE. Medicaid expansion produces long-term impact on insurance coverage rates in community health centers. J Prim Care Community Health. 2017;8(4):206-212.
10. Sessions K, Hassan A, McLeod TG, Wieland ML. Health insurance status and eligibility among patients who seek healthcare at a free clinic in the Affordable Care Act era. J Community Health. 2018; 43(2):263-267.

11. Cole MB, Trivedi AN, Wright B, Carey K. Health insurance coverage and access to care for community health center patients: evidence following the Affordable Care Act. J Gen Intern Med. 2018;33(9): 1444-1446.

12. DeVoe JE, Gold R, Cottrell E, et al. The ADVANCE network: accelerating data value across a national community health center network. J Am Med Inform Assoc. 2014;21(4):591-595.

13. Brooks T, Wagnerman K, Artiga S, Cornachione E, Ubri P. Medicaid and CHIP Eligibility, Enrollment, Renewal, and Cost Sharing Policies as of January 2017: Findings From a 50-State Survey. Henry J. Kaiser Family Foundation: Menlo Park, CA; Jan 2017.

14. Centers for Medicare and Medicaid Services. Benefits. https://www. medicaid.gov/medicaid/benefits/index.html. Accessed Nov 2, 2018.

15. Marino M, Angier $H$, Valenzuela $S$, et al. Medicaid coverage accuracy in electronic health records. Prev Med Rep. 2018;11:297-304.

16. Hatch B, Tillotson C, Angier $\mathrm{H}$, et al. Using the electronic health record for assessment of health insurance in community health centers. J Am Med Inform Assoc. 2016;23(5):984-990.

17. Bailey SR, Hoopes MJ, Marino M, et al. Effect of gaining insurance coverage on smoking cessation in community health centers: a cohort study. J Gen Intern Med. 2016;31(10):1198-1205.

18. Tsai CL, Griswold SK, Clark S, Camargo CA Jr. Factors associated with frequency of emergency department visits for chronic obstructive pulmonary disease exacerbation. J Gen Intern Med. 2007;22(6): 799-804.

19. Charlson ME, Charlson RE, Peterson JC, Marinopoulos SS, Briggs WM, Hollenberg JP. The Charlson comorbidity index is adapted to predict costs of chronic disease in primary care patients. J Clin Epidemiol. 2008;61(12):1234-1240.

20. Claxton G, Cox C, Daminco A, Levitt L, Pollitz K; Henry J. Kaiser Family Foundation. Pre-existing conditions and medical underwriting in the individual insurance market prior to the ACA. http://files. kff.org/attachment/Issue-Brief-Pre-existing-Conditions-and-MedicalUnderwriting-in-the-Individual-Insurance-Market-Prior-to-the-ACA. Published Dec 2016. Accessed Jun 26, 2017.

21. DiJulio B, Firth J, Brodie M. Kaiser Health tracking poll: December 2015. https://www.kff.org/health-costs/poll-finding/kaiser-healthtracking-poll-december-2015/. Published Dec 17, 2015. Accessed Nov 2, 2018.

22. Emson PC, Shoham S, Feler C, Buss T, Price J, Wilson CJ. The use of a retroviral vector to identify foetal striatal neurones transplanted into the adult striatum. Exp Brain Res. 1990;79(2):427-430.

23. Ghaddar S, Byun J, Krishnaswami J. Health insurance literacy and awareness of the Affordable Care Act in a vulnerable Hispanic population. Patient Educ Couns. 2018;101(12):2233-2240.

24. Gold R, Bailey SR, O'Malley JP, et al. Estimating demand for care after a medicaid expansion: lessons from Oregon. J Ambul Care Manage. 2014;37(4):282-292.

25. Polsky D, Richards M, Basseyn S, et al. Appointment availability after increases in Medicaid payments for primary care. $N$ Engl J Med. 2015;372(6):537-545. 